\title{
Construction of a model to predict the prognosis of patients with cholangiocarcinoma using alternative splicing events
}

\author{
HUA-YU WU ${ }^{1,2^{*}}$, YI WEI ${ }^{1 *}$, LI-MIN LIU ${ }^{3}$, ZHONG-BIAO CHEN $^{4}$, QI-PING HU ${ }^{2}$ and SHANG-LING PAN ${ }^{1}$ \\ Departments of ${ }^{1}$ Pathophysiology and ${ }^{2}$ Cell Biology and Genetics, School of Pre-Clinical Medicine, \\ ${ }^{3}$ Department of Toxicology, College of Pharmacy, Guangxi Medical University, Nanning, Guangxi 530021; \\ ${ }^{4}$ Department of General Surgery, The First People's Hospital of Yulin, Yulin, Guangxi 537000, P.R. China
}

Received February 8, 2019; Accepted August 13, 2019

DOI: $10.3892 / 01.2019 .10838$

\begin{abstract}
Cholangiocarcinoma (CCA) is a type of malignant tumor that originates in the mucosal epithelial cells of the biliary system. It is a highly aggressive cancer that progresses rapidly, has low surgical resection rates and a high recurrence. At present, no prognostic molecular biomarker for CCA has been identified. However, CCA progression is affected by mRNA precursors that modify gene expression levels and protein structures through alternative splicing (AS) events, which create molecular indicators that may potentially be used to predict CCA outcomes. The present study aimed to construct a model to predict CCA prognosis based on AS events. Using prognostic data available from The Cancer Genome Atlas, including the percent spliced index of AS events obtained from TCGASpliceSeq in 32 CCA cases, univariate and multivariate Cox regression analyses were performed to assess the associations between AS events and the overall survival (OS) rates of patients with CCA. Additional multivariate Cox regression analyses were used to identify AS events that were
\end{abstract}

Correspondence to: Professor Shang-Ling Pan, Department of Pathophysiology, School of Pre-Clinical Medicine, Guangxi Medical University, 22 Shuangyong Road, Nanning, Guangxi 530021, P.R. China E-mail: slpan@gxmu.edu.cn

Professor Qi-Ping Hu, Department of Cell Biology and Genetics, School of Pre-Clinical Medicine, Guangxi Medical University, 22 Shuangyong Road, Nanning, Guangxi 530021, P.R. China

E-mail: huqiping@gxmu.edu.cn

*Contributed equally

Abbreviations: CCA, cholangiocarcinoma; TCGA, The Cancer Genome Atlas; K-M, Kaplan-Meier; AUC, area under the curve; ROC, receiver operator characteristic; PSI, percent spliced index; ES, exon skip; ME, mutually exclusive exons; RI, retained intron; $\mathrm{AP}$, alternate promoter; AT, alternate terminator; $\mathrm{AD}$, alternate donor site; AA, alternate acceptor site; GO, Gene Ontology; KEGG, Kyoto Encyclopedia of Genes and Genomes

Key words: splicing, TCGASpliceSeq, cholangiocarcinoma, The Cancer Genome Atlas, prognosis significantly associated with prognosis, which were used to construct a prediction model with a prognostic index (PI). A receiver operating characteristic (ROC) curve was used to determine the predictive value of the PI, and Pearson's correlation analysis was used to determine the association between OS-related AS events and splicing factors. A total of 38,804 AS events were identified in 9,673 CCA genes, among which univariate Cox regression analysis identified 1,639 AS events associated with OS $(\mathrm{P}<0.05)$; multivariate Cox regression analysis narrowed this list to $23 \mathrm{CCA}$ AS events $(\mathrm{P}<0.001)$. The final PI model was constructed to predict the survival of patients with CCA; the ROC curve demonstrated that it had a high predictive power for CCA prognosis, with a highest area under the curve of 0.986 . Correlations between 23 OS-related AS events and splicing factors were also noted, and may thus, these AS events may be used to improve predictions of OS. In conclusion, AS events exhibited potential for predicting the prognosis of patients with CCA, and thus, the effects of AS events in CCA required further examination.

\section{Introduction}

Cholangiocarcinoma (CCA) is a malignant tumor that originates in the mucosal epithelial cells of the biliary system. With a high degree of malignancy, rapid progression, low surgical resection rates and high recurrence rates, CCA prognoses are unsatisfactory (1-4). Recent studies of the molecular pathological mechanisms of CCA have demonstrated that tumor formation, growth, invasion, metastasis and other processes in CCA are regulated by a variety of molecules and signal transduction pathways, such as the aPKC-ı/P-Sp1/Snail signaling pathway and the Merlin/YAP/c-Myc/mTOR signaling pathway $(5,6)$. This information has assisted early diagnosis, prognosis predictions and drug development. However, due to the complexity of CCA development, there are currently no specific biomarkers that meet clinical standards (7-10). Therefore, there is an urgent need to identify suitable biomolecular markers that can screen for CCA and assist in prognostic evaluations.

Eukaryotic mRNA precursors are known to remove certain unused fragments during the maturation process of mRNAs, allowing the remaining fragments to be rejoined. The fragments that are removed or left in place vary in their 
effects, depending on the cell type and status. Thus, a gene can encode multiple proteins in a process called alternative splicing (AS) $(11,12)$. Previous studies have demonstrated that AS events occur ubiquitously in eukaryotes, with $>95 \%$ of genes undergoing AS events (13-15). However, AS events are more common in cancer cells and affect cancer development and treatment resistance (16-24). In addition, AS events in tumor cells may be used as molecular markers that can differentially diagnose tumor types and predict prognosis (25-28), as well as serve as potential targets for cancer treatment (29-33). Application of AS events as a means diagnosing and predicting prognosis has been demonstrated in in prostate adenocarcinoma, uteri corpus endometrial carcinoma and colorectal cancer (34-36).

CCA involves a high frequency of gene mutations and abnormal epigenetic changes, including DNA methylation, and histone and RNA modifications (37), all of which may lead to the occurrence of AS events (19,38-47). AS events modify gene expression and influence protein structure by changing coding regions, thus regulating certain biological processes. For example, the widely reported tumor suppressor genes tumor protein $\mathrm{p} 53$, AT-rich interaction domain $1 \mathrm{~A}$, $P T E N$ and $P I 3 K$, as well as the proto-oncogenes NOTCHI and MET proto-oncogene, receptor tyrosine kinase, cause variations in gene function through AS events, thus affecting cancer development (30). Similarly, the apoptosis-related genes $B C L-X$ and modulator of VRAC current 1 have been demonstrated to serve opposite functions in promoting and resisting apoptosis due to the occurrence of AS events (48-51). AS can also alter the amino acid sequence of a protein, which may destroy the target for certain antitumor drugs and result in drug resistance $(20,30,52)$. AS events therefore serve a unique role in the diagnosis and treatment of tumors.

Previous studies on the association between AS events and tumors have focused on the single-gene level, and few large-scale data mining studies based on high-throughput sequencing exist (53-55). The Cancer Genome Atlas (TCGA) database (portal.gdc.cancer.gov/) contains high-throughput sequencing data and comprehensive clinical information from a large number of cancer samples. Previous studies using TCGA data have reported that AS events may be used as prognostic indicators for lung (56), ovarian (57), bladder (58) cancer and gastrointestinal pan-adenocarcinomas $(59,60)$. However, the prognostic value of AS events has not yet been reported for CCA. In the present study, the overall survival (OS)-related AS events in CCA were systemically evaluated. These findings may facilitate development of novel genomic models for clinical cancer management, and construction of novel models based on the prognostic index (PI) to predict CCA survival.

\section{Materials and methods}

Data collection. The percent spliced index (PSI) values for CCA AS events were downloaded from the TCGASpliceSeq database (http://bioinformatics.mdanderson. org/TCGASpliceSeq/) (61). PSI is the ratio of normalized read counts indicating inclusion of a transcript element to the total normalized read counts for that event (i.e., both inclusion and exclusion read counts). PSI values range from 0 to 1 , indicating the likelihood of the existence of an exon. Information related to seven types of AS events: Exon skip (ES), mutually exclusive exons (ME), retained intron (RI), alternate promoter (AP), alternate terminator (AT), alternate donor (AD) site and alternate acceptor (AA) site events, was included in the analysis. Corresponding clinical information and gene expression levels of the samples were obtained from TCGA database. The database included data from 32 patients with CCA with complete clinical information and total survival time $>90$ days. Of the 32 patients with CCA, 13 were male and 19 were female. The age of the patients was 29-82, and the median age was 66.5 , with 27 patients older than 50 and 5 patients younger than 50 .

Association between AS events and survival. $\mathrm{R}$ software ( $\mathrm{R}$ version 3.4.2) (62) was used to perform univariate Cox regression analysis to investigate the PSI prognostic values from the seven types of AS events and the differentially expressed genes in the 32 CCA cases. AS events with $\mathrm{P}<0.05$ were selected for further analysis. The event-dependent survival curve for the top three AS events was plotted (collating P-values from low to high).

Systematic review. To confirm that the identified AS events were CCA-specific, all prognostic AS events from different types of cancer were extracted from published articles on PubMed (https://www.ncbi.nlm.nih.gov/), Wiley Online Library (https://onlinelibrary.wiley.com/), EBSCO (https://www. ebsco.com/), Web of Science (https://www.webofknowledge. com/), and Google Scholar (https://scholar.google.com/) on or prior to 1st May 2019 for comparison. The key words were as follows: 'cancer OR carcinoma OR adenocarcinoma OR tumour OR tumor OR malignanc* OR neoplas*' AND 'prognostic OR prognosis OR predict*' AND ‘ 'alternative splicing' OR AS'. The studies which were included needed to meet the following criteria: i) Studies describing cancer prognosis with a predicted model constructed using prognostic AS events; and ii) the prognostic AS events could be extracted from the studies. All the included studies were assessed using the bias assessment for studies of diagnostic accuracy (QUADAS) guidelines (63) and had a QUADAS score of $\leq 7$. The following data were extracted from the included studies: First author's name, year of publication and prognostic AS events.

AS event interaction analysis and gene network construction. The interactions of the seven types of AS events were analyzed using the UpSetR package for R (64), and an UpSet plot was drawn. The network was constructed using the Reactome FI plug-in (65) for Cytoscape 3.6.0 (66), with AS events identified using univariate Cox regression analysis $(\mathrm{P}<0.01)$. Genes with AS events were processed using the Kyoto Encyclopedia of Genes and Genomes (KEGG) and Gene Ontology (GO) gene enrichment analyses. Due to the occurrence of a large number of significant GO pathways, only the top five pathways per group were presented using the ggplot2 package for $\mathrm{R}(67)$.

PI construction and prognostic value assessment. To assess the association between AS events and OS, univariate Cox regression was carried out with a significance threshold of $\mathrm{P}<0.05$. The top ten most promising AS events, based on 
their prognostic value, AS events selected for each splicing type. Multivariate Cox regression analysis was performed, splicing events with $\mathrm{P}<0.05$ were selected as PIs. The model was established by grouping together all splicing events of the seven splicing event types. The samples were divided into two groups according to the median PSI value of the splicing events. The formula used to calculate the PI was as follows:

$$
\text { Risk score }=\sum_{i}^{n} \text { PSI } i * \beta i
$$

The clinical prognostic value of the PI was assessed using Kaplan-Meier (K-M) curve analysis and time-dependent receiver operator characteristic (ROC) curve analysis. The time-dependent ROC curve was drawn using the survival ROC package for $\mathrm{R}(68)$.

Experimental validation of AS events in clinical CCA tissue samples. In addition to the aforementioned analyses, the existence of the AS events predicted by the TCGASpliceSeq database was verified using PCR analyses with six samples of CCA tumor tissues separately collected from a 37-year-old male patient and a 38-year-old female patient during surgery at the Pathology Department of the First Affiliated Hospital of Guangxi Medical University (Nanning, China) between February 2017 and October 2017. The study was approved by the Ethics Committee of the First Affiliated Hospital of Guangxi Medical University, and written informed consent was obtained from all patients. Total RNA was extracted using the AxyPrep Multisource Total RNA Miniprep kit (Axygen; Corning, Inc.), quantified using a NanoDrop 2000 (Thermo Fisher Scientific, Inc.) and $500 \mathrm{ng}$ total RNA was reverse-transcribed into cDNA using MiScript $^{\circledR}$ II RT SuperMix kit (Vazyme). AS events in phytanoyl-CoA 2-hydroxylase (PHYH)_100582_ES and transferrin receptor 2 (TFR2)_80979_ES were selected for verification. The mean PSI of PHYH_100582_ES in TCGASpliceSeq database was 0.78 , and ES was present in exons 7 and 8 . The forward primer (primer 1) was designed based on exon 6, and the sequence was 5'-GATACTGCACTC TCCCCGAG-3'. The reverse primer (primer 2) was designed based on exon 9, and the primer sequence was 5'-GACCAG ATCCGTGGATGAGC-3'. The PCR system contained $10 \mu \mathrm{l}$ 2X PCR Master mix (Thermo Fisher Scientific, Inc), $1 \mu 1$ each of the forward and reverse primers $(10 \mu \mathrm{M}), 1 \mu \mathrm{l}$ cDNA and $7 \mu \mathrm{l}$ nuclease-free water (total volume, $20 \mu \mathrm{l}$ ). The thermocycling conditions were as follows: $95^{\circ} \mathrm{C}$ for $3 \mathrm{~min}$, followed by 35 cycles of $95^{\circ} \mathrm{C}$ for $30 \mathrm{sec}, 60^{\circ} \mathrm{C}$ for $30 \mathrm{sec}$ and $72^{\circ} \mathrm{C}$ for $1 \mathrm{~min}$. PCR products were subjected to electrophoresis on $2 \%$ agarose gels. The mean PSI of TFR2_80979_ES in the TCGASpliceSeq database was 1, and ES was present in exon 10. The forward primer (primer 3) was designed based on exon 9 , and the primer sequence was 5'-CAGCCCATCAGTGCA GACAT-3'. The reverse primer (primer 4) was designed based on exon 11, and the primer sequence was 5'-TTGTTGACC ACTAGCCGCAG-3'. The PCR system and thermocycling conditions were as mentioned above.

Correlation analysis between splicing factors and prognosis-related AS events. A splicing factor that serves as a splicing activator when bound to an intronic enhancer element may function as a repressor when bound to its splicing element in

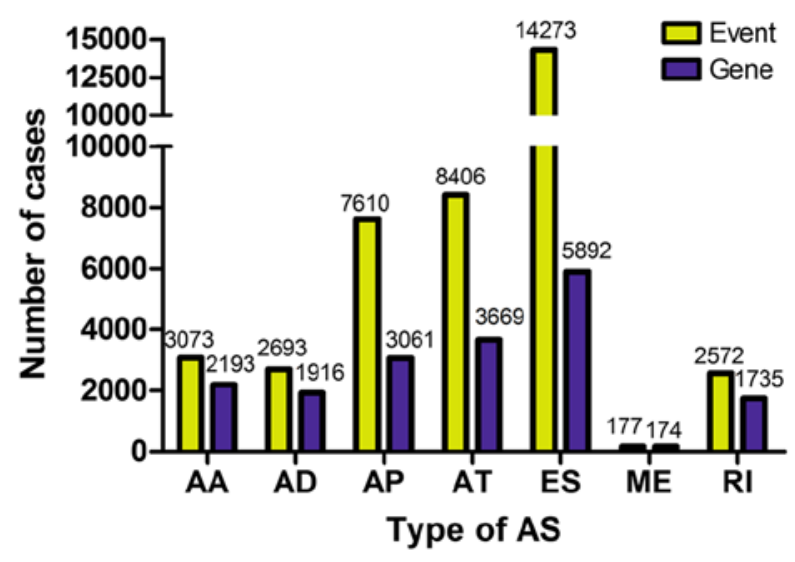

Figure 1. AS events and the numbers of related genes associated with cholangiocarcinoma. AA, alternate acceptor site; AD, alternate donor site; AP, alternate promoter; AT, alternate terminator; ES, exon skip; ME, mutually exclusive exons; RI, retained intron; AS, alternative splicing.

the context of an exon (69). Therefore, the correlations between splicing factors and the prognosis-related AS events were investigated. Splicing factor information was obtained from the SpliceAid 2 database (http://193.206.120.249/splicing_tissue. html) (70). Univariate Cox regression analysis was performed on the splicing events and splicing factors, followed by Pearson's correlation analysis between the significant splicing events, splicing factors and the OS-related AS events identified by multivariate Cox analysis. The results were visualized using Cytoscape version 3.4.0 (66).

\section{Results}

CCA AS events. A total of 38,804 AS events were obtained from 9,673 genes in 32 CCA cases from TCGA SpliceSeq dataset. The numbers of each type of AS event and the corresponding genes are presented in Fig. 1. The results demonstrated that a single gene may undergo multiple AS events simultaneously. ES was the most frequent AS event in this dataset.

AS events associated with CCA OS rates. Univariate Cox analysis revealed that 1,639 AS events were associated with CCA OS rates $(\mathrm{P}<0.05)$ and that certain genes had undergone multiple OS-related AS events. Visualization of the intersecting sets was performed using UpSet plotting software (Fig. 2), and $\leq 3$ AS events were identified to be associated with OS rates within the same gene. KEGG pathway enrichment analysis revealed that the genes corresponding to these AS events were primarily enriched in 'biosynthesis of antibiotics', 'axon guidance', 'pancreatic cancer', 'RAP1 signaling pathway' and 'SNARE interactions in vesicular transport' (Fig. 3A). GO gene enrichment analysis demonstrated that the genes with OS-related AS events corresponded to 'protein transport', 'cell-cell adhesion', 'apoptotic process', 'protein localization to the nucleus' and 'positive regulation of transcription from RNA polymerase II promoters' (Fig. 3B). Using a network map of these genes, MYC associated factor $\mathrm{X}(M A X)$, mitogen-activated protein kinase 11 (MAPK11), $\gamma$-aminobutyric acid type A receptor-associated protein-like 1 (GABARAPL1), checkpoint kinase 1 (CHEK1), X-linked 


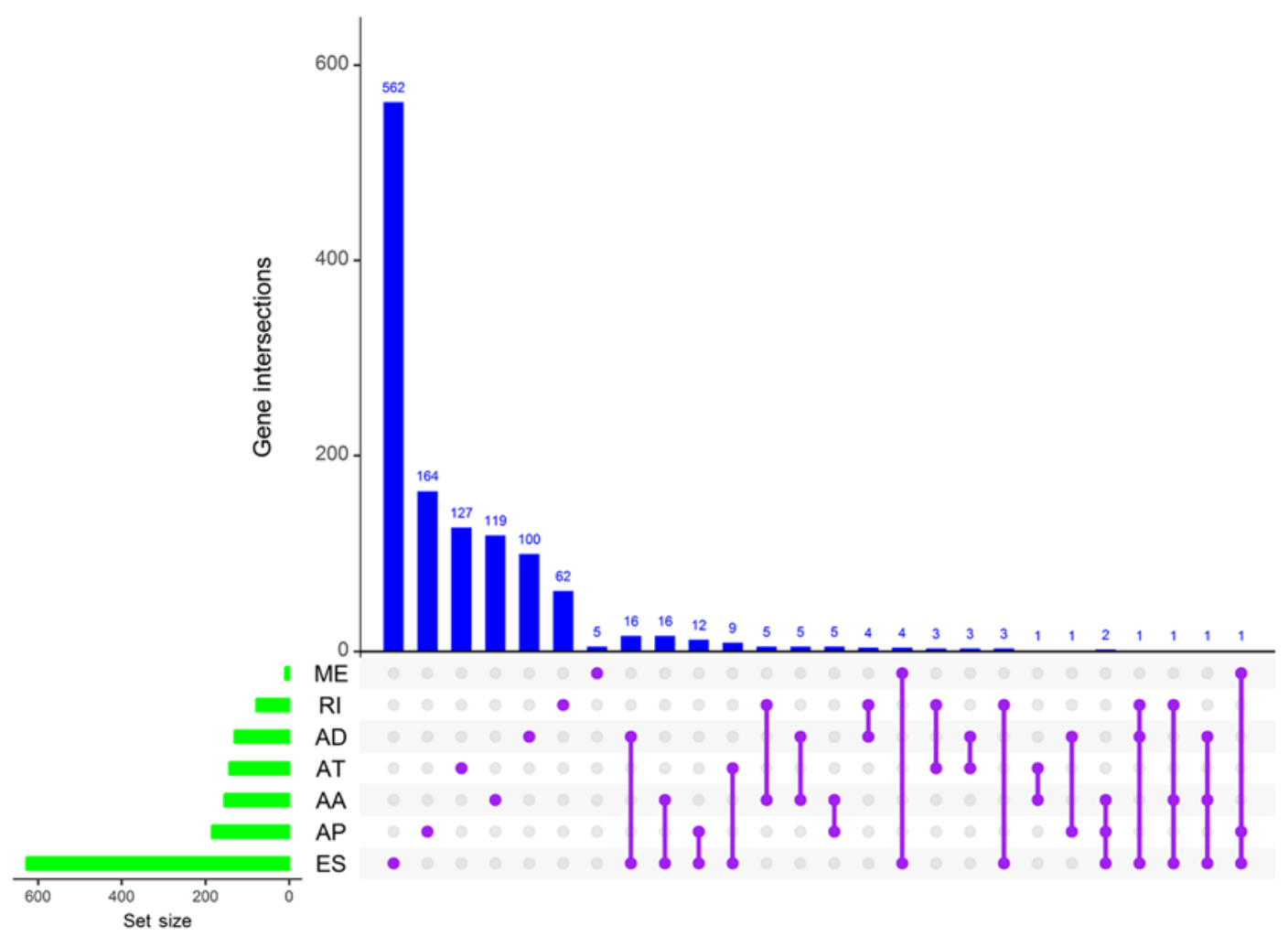

Figure 2. UpSet plot of survival-related alternative splicing events in cholangiocarcinoma. A single gene can undergo up to three simultaneous prognosis-related alternative splicing events. AA, alternate acceptor site; AD, alternate donor site; AP, alternate promoter; AT, alternate terminator; ES, exon skip; ME, mutually exclusive exons; RI, retained intron.

inhibitor of apoptosis (XIAP) and forkhead box A1 (FOXAl) exhibited the highest degrees of connectivity and were thus considered critical positions of the network (Fig. 4).

OS-related AS events are CCA-specific. Comparisons with other types of cancer demonstrated that ME-GTF2H3-306194 occurred in papillary thyroid cancer and CCA, whereas the other identified AS events were CCA-specific (Table I).

AS events that may be used as CCA prognostic indicators. The three most statistically significant AS events were selected, and their gene expression levels were used to calculate their prognostic values in CCA (Fig. 5). K-M analysis revealed that these prognostic signatures significantly separated patients with distinct prognoses. To assess the ability of these splicing events to predict CCA prognosis, a time-dependent ROC curve was established. The results of the ROC curve suggested that a single indicator was not enough to predict patient prognosis, as the area under the curve (AUC) values were low for the AS events and gene expression values (Fig. 5). Therefore, ten AS events with the most significant prognostic values in each splicing type were selected to construct the PIs (Table II). The results suggested that these PIs had higher predictive values compared with single indicators (Fig. 6). The AUCs for each of the seven subtypes of AS were as follows: AA, 0.901; AD, 0.858; AP, 0.986; AT, 0.839; ES, 0.939; ME, 0.779; and RI, 0.859. In addition, the ten most significant AS events from all types combined were selected to construct a final prognostic model; the AUC was 0.984 (Fig. 6). It is worth noting that the predictive efficacy of the prognostic model with seven combined splicing events (0.984) was slightly lower than that obtained with AP alone (0.986).

Confirmation of PHYH_100582_ES and TFR2_80979_ES by clinical samples. Towards PHYH_100582_ES, the PCR products were predicted to comprise two bands; the band without ES was predicted to be $414 \mathrm{bp}$ in length, whereas the band size following ES was predicted to be $144 \mathrm{bp}$ in length (Fig. 7A). If the TFR2_80979_ES event did not occur, the PCR product was predicted to be $238 \mathrm{bp}$; following the ES event, the PCR product size was predicted to be $140 \mathrm{bp}$ (Fig. 7B). Since the PSI value of this ES event was 1, the ES event occurred in all TFR2 mRNAs; if the TCGASpliceSeq database was accurate, the PCR product was predicted to be a single band. The results of PHYH_100582_ES and TFR2_80979_ES were consistent with those predicted by the TCGASpliceSeq database. The PCR products of PHYH_100582_ES were 414 and 144 bp (Fig. 7C), suggesting that, in CCA tissues, a proportion of PHYH mRNA had undergone an ES event. The PSI of TFR2_80979_ES in the TCGASpliceSeq database was 1, indicating that ES events occurred in all TFR2 mRNAs. The electrophoresis result demonstrated that the product of TFR2_80979_ES was a single band of $140 \mathrm{bp}$; no band of 238 bp was observed (Fig. 7D), suggesting that ES events occurred in all TFR2 mRNA. These results were consistent with those suggested by the RNA sequencing data from TCGASpliceSeq.

Correlation between splicing factors and prognosis-related $A S$ events. A total of $11 \mathrm{AS}$ events and corresponding splicing 
A
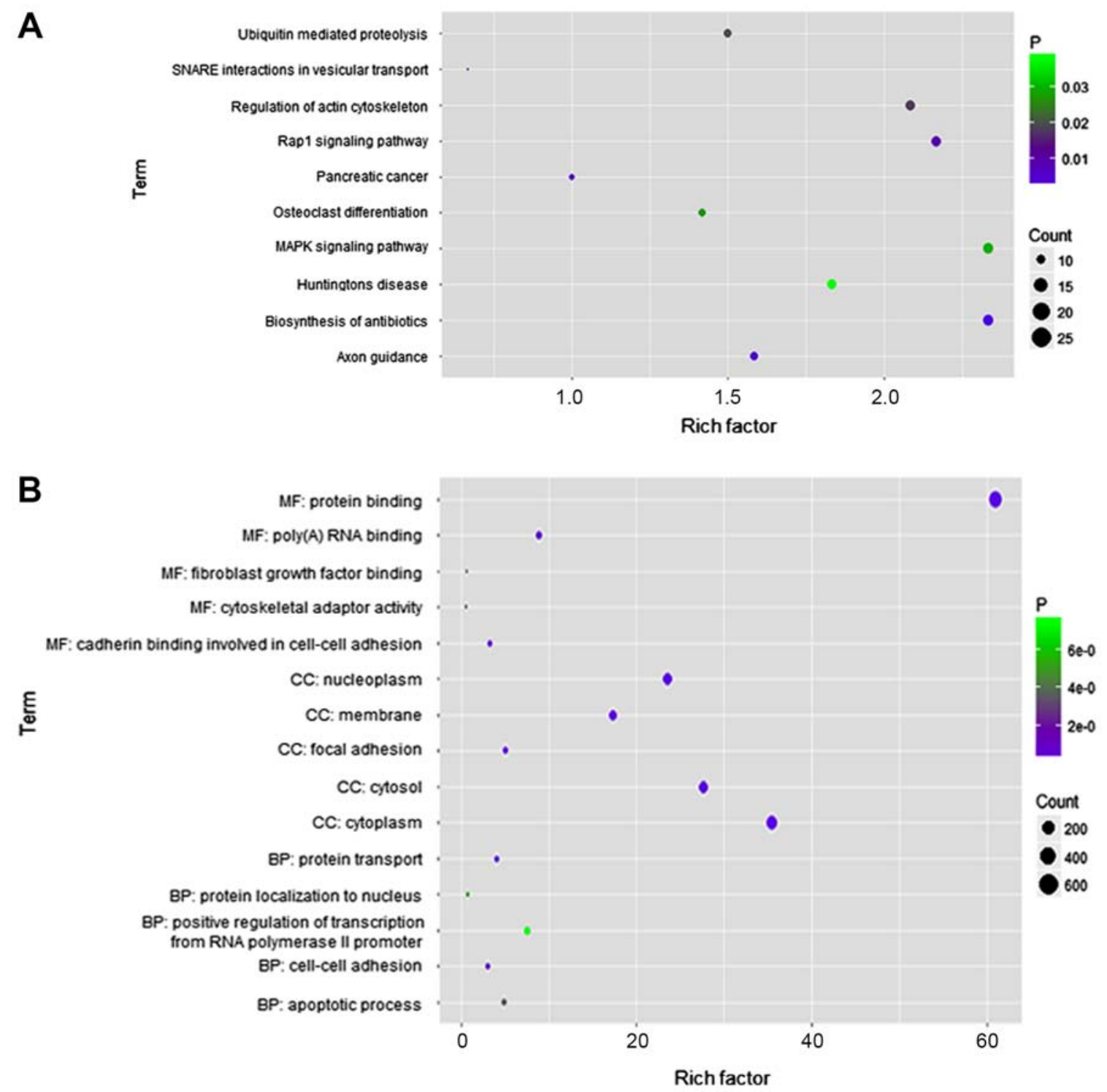

Figure 3. KEGG pathway and GO analysis of genes with overall survival-related alternative splicing in cholangiocarcinoma (A) KEGG pathway analysis of genes with overall survival-associated alternative splicing events. (B) GO enrichment analysis of genes with overall survival-associated alternative splicing events. Rich factor, ratio of differentially expressed gene numbers annotated in this term to all gene numbers annotated in this term.; KEGG, Kyoto Encyclopedia of Genes and Genomes; GO, Gene Ontology; BP, biological process; CC, cellular component; MF, molecular function.

factors significantly related to CCA OS rates $(\mathrm{P}<0.05)$ were identified. Multivariate Cox analysis identified 23 prognosis-related AS events (Table III; P<0.001); 17 of these exhibited positive correlations with OS, whereas 6 exhibited negative correlations with OS. Of these 23 AS events, 15 were correlated with a group of splicing factors, the expression of which was directly associated with AS events $(|\mathrm{r}|>0.3$; $\mathrm{P}<0.05)$. AS events are primarily regulated by splicing factors, which often bind to pre-mRNAs and regulate RNA splicing by influencing exon selection and splicing site. Therefore, the associations between survival-related AS events and splicing factors were determined (Fig. 8).

\section{Discussion}

Using data mined from TCGASpliceSeq and in silico approaches, the present study identified that a number of AS events are closely associated with survival in CCA. The present study is the first to report this type of result. The results of the present study also demonstrated that AS events may be used to construct a PI model that effectively determines CCA prognosis.

The incidence of CCA has increased in recent years. The prognosis of advanced CCA is poor, with an extremely low 5-year survival rate (7). The prognosis depends on the synergistic effects of various factors; however, no clear and effective molecular markers have been identified for CCA diagnosis and treatment. Carcinoembryonic antigens (CEAs) and carbohydrate antigen (CA) 199, CA 125, CA 50 and CA 242 are currently used as CCA tumor markers, but these biomarkers have disadvantages in clinical application. For example, the majority of studies that examined CA 199 as a biomarker for the detection of CCA have reported its suboptimal accuracy, with wide variation in reported sensitivity (38-93\%) and specificity (67-98\%) (71). CA 125 is upregulated in $65 \%$ of patients with CCA and is of value in predicting survival (72); 


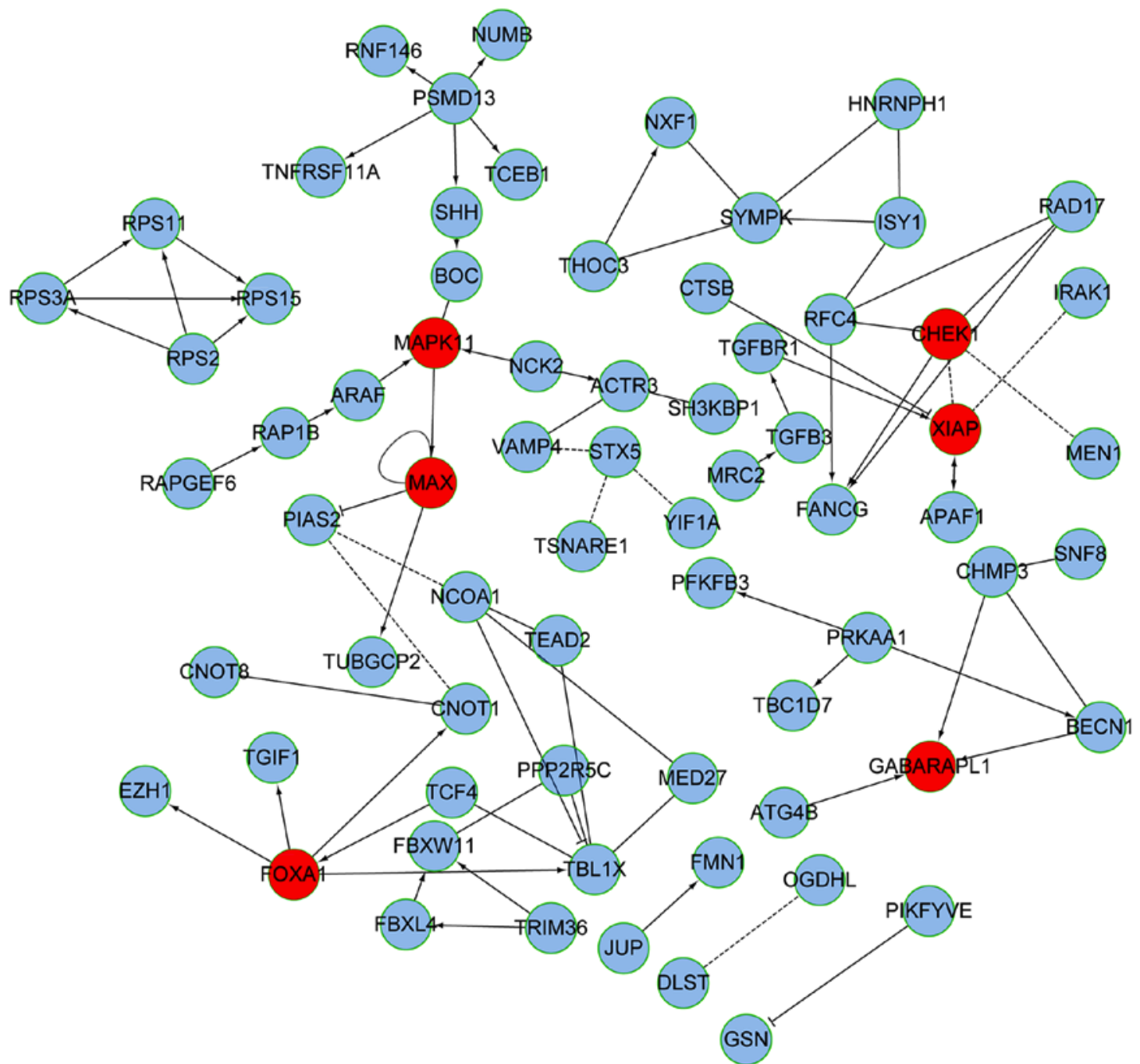

Figure 4. A network diagram of overall survival-related alternative splicing events in cholangiocarcinoma. Genes corresponding to alternative splicing events were identified and a network map was constructed using Cytoscape software. The genes with the highest degree of connectivity are marked in red.
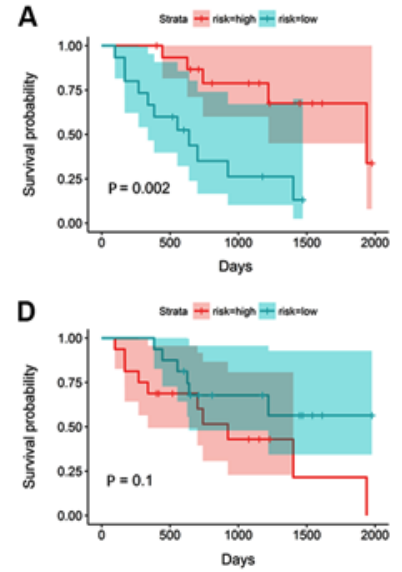
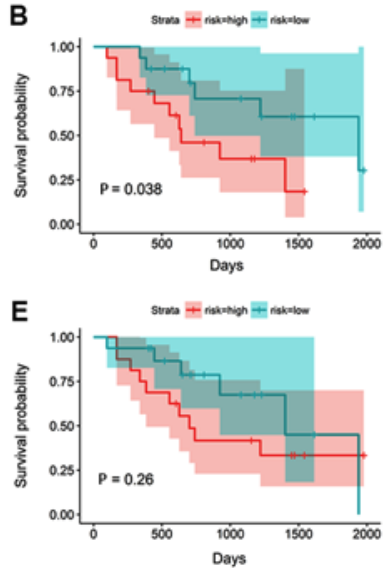
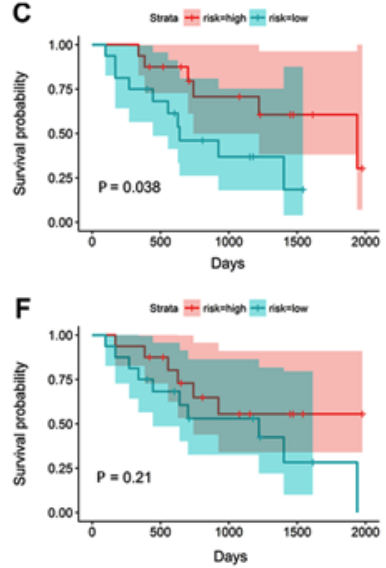

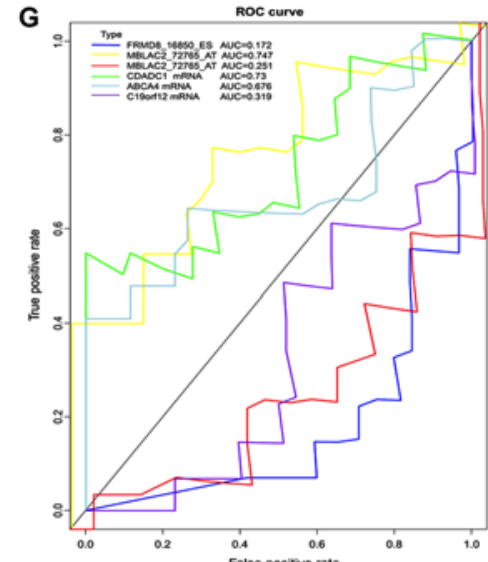

Figure 5. Kaplan-Meier survival and ROC curves of alternative splicing events and gene expression of the top three factors identified using univariate Cox analysis. Blue, low-risk group; red, high-risk group. (A-C) Kaplan-Meier curves of alternative splicing events. (A) FRMD8_16850_ES (HR, 0.736; 95\% CI, 0.627-0.824; P<0.001). (B) MBLAC2_72765_AT (HR, 1.186; 95\% CI, 1.084-1.298; P<0.001). (C) MBLAC2_72766_AT (HR, 0.843; 95\% CI, 0.771-0.923; P<0.001). (D-F) Kaplan-Meier curves of gene expression levels. (D) CDADC1 (HR, 1060.208; 95\% CI, 15.259-73662.548; P=0.001). (E) ABCA4 (HR, 13.302; 95\% CI, 2.596-68.156; P=0.002). (F) C19orf12 (HR, 0.0004; 95\%CI, 0.000001-0.081; P=0.004). (G) ROC curves of alternative splicing events and gene expression levels: FRMD8_16850_ES, MBLAC2_72765_AT, MBLAC2_72766_AT, CDADC1, ABCA4 and C19orf12. AA, alternate acceptor; AD, alternate donor; AP, alternate promoter; AT, alternate terminator; ROC, receiver operating characteristic; AUC, area under the curve; ES, exon skip; ME, mutually exclusive exon; RI, retained intron; HR, Hazard ratio; CI, confidence interval; FRMD, FERM domain-containing 8; MBLAC2, metallo- $\beta$-lactamase domain-containing 2; CDADC1, cytidine and DCMP deaminase domain-containing 1; ABCA4, ATP-binding cassette subfamily A member 4; C19orf12, chromosome 19 open reading frame 12. 

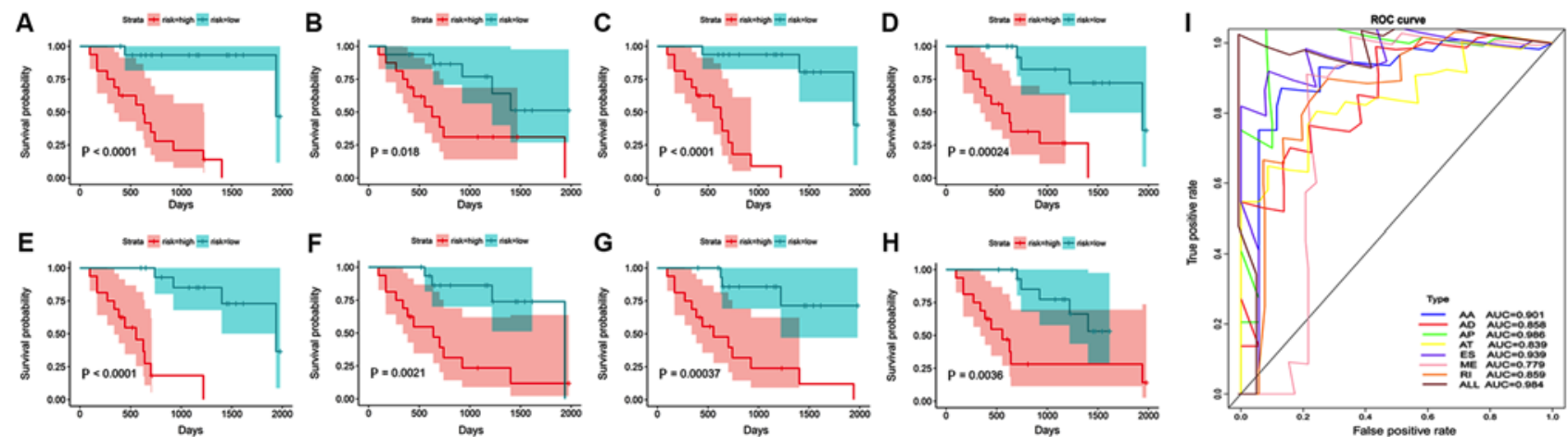

Figure 6. Kaplan-Meier curves based on the prognostic index and its ROC curves. Blue, low-risk group; red, high-risk group. (A-H) Kaplan-Meier curves of alternative splicing events. (A) AA site. (B) AD site. (C) AP. (D) AT. (E) ES. (F) ME exons. (G) RI. (H) ALL. (I) ROC curves of AA, AD, AP, AT, ES, ME, RI and ALL. AA, alternate acceptor; AD, alternate donor; AP, alternate promoter; AT, alternate terminator; ROC, receiver operating characteristic; AUC, area under the curve; ES, exon skip; ME, mutually exclusive exon; RI, retained intron; ALL, all alternative splicing types combined.

A

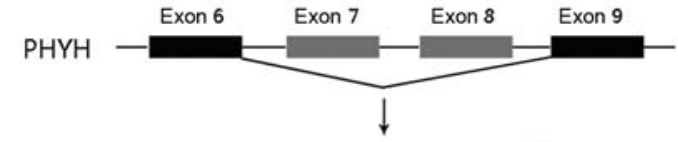

None ES

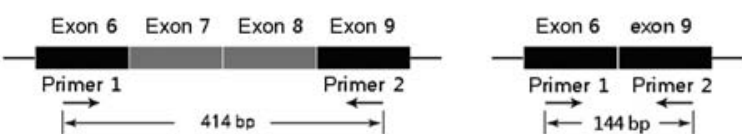

C

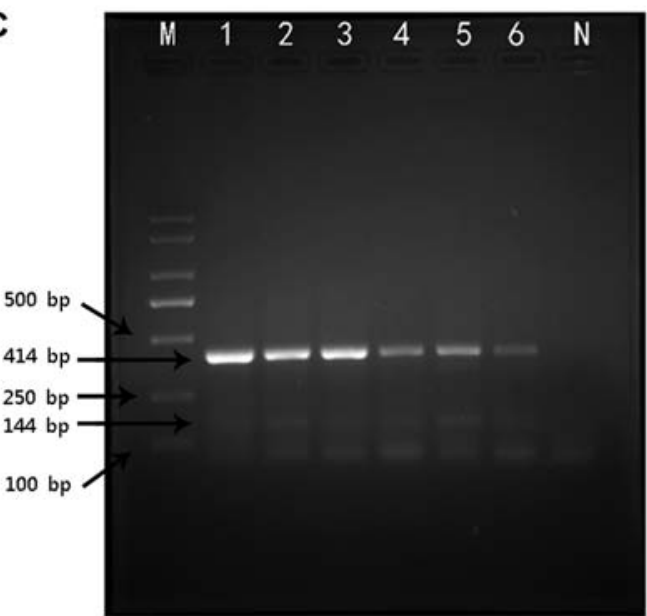

B

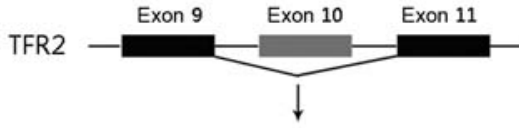

None ES

ES

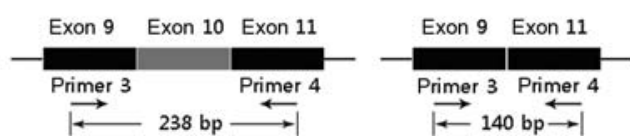

D

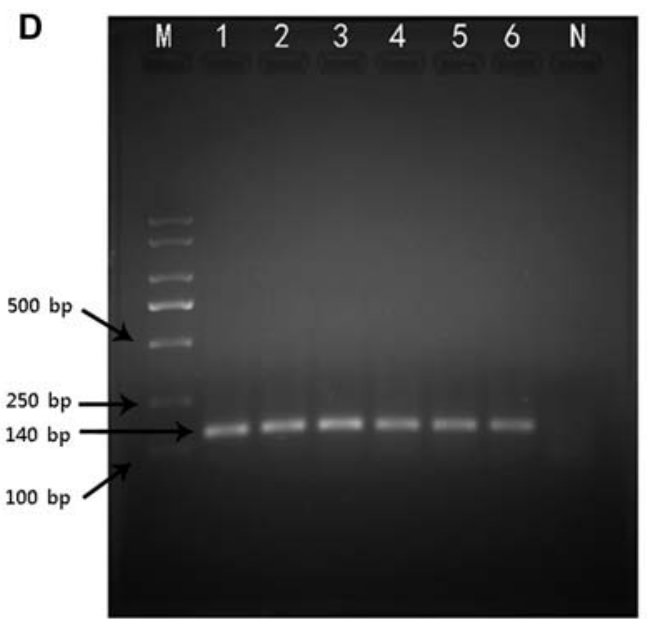

Figure 7. Validation of predicted AS events in clinical samples. (A) Illustration of PHYH_100582_ES AS event in the present study. Following the ES event, exons 7 and 8 of PHYH mRNA were cut out, and exons 6 and 9 were directly spliced. (B) Illustration of TFR2_80979_ES AS event in the present study. Following the ES event, exon 10 of TFR2 mRNA was cut out, and exons 9 and 11 exons were directly spliced. (C) PHYH_100582_ES event electropherogram. Lanes 1-6 demonstrate the PCR amplification results of cDNA from six CCA tissues with bands of 414 bp and 144 bp. (D) TFR2_80979_ES event electropherogram. Lanes 1-6 demonstrate the PCR amplification results of cDNA from six CCA tissues with a single band of 140 bp. M, DNA marker; N, negative control without a template; AS, alternative splicing; ES, exon skip; PHYH, phytanoyl-CoA 2-hydroxylase; TRF2, transferrin receptor 2; CCA, cholangiocarcinoma.

however, there are no further studies on CA 125 in CCA. CA 50 exhibits cross-antigenicity with CA 199, and elevated serum CA 50 levels are commonly used to diagnose or prognose pancreatic and colorectal cancers (73). However, Shan et al (74) demonstrated that elevated serum CA 50 levels are not always associated with the expression of CA 50 in cancer tissues. Despite the clinical utility of CA 242, it is not sufficiently effective in the early detection of cancer, since elevated classical tumor biomarker levels indicate the presence of a significant number of cancer cells (75).
At the genetic level, a number of genes are abnormally expressed in CCA. For example, the abnormal expression of genes such as transforming growth factor $\beta 1, S M A D 4, c-M E T$, matrix metallopeptidase 7, vascular endothelial growth factor (VEGF)-A, VEGF-B, VEGF-C and VEGF-D are associated with poor prognosis (76-79). Mutations in genes such as human epidermal growth factor receptor 2, TP53, KRAS, cytosolic NADP-dependent isocitrate dehydrogenase and mitochondrial NADP-dependent isocitrate dehydrogenase can also affect the prognosis of patients with CCA, but the prognostic value of 
Table I. Prognostic alternative splicing events in different types of cancer.

\begin{tabular}{llll}
\hline $\begin{array}{l}\text { Author, } \\
\text { year }\end{array}$ & Type & PMID & Alternative splicing events \\
\hline
\end{tabular}

Lin et al, Papillary thyroid 2019 cancer

Gao et al, Uteri corpus 2019 endometrial carcinoma

$\begin{array}{ll}\begin{array}{l}\text { Huang } \\ \text { et al, }\end{array} & \begin{array}{l}\text { Prostate } \\ \text { adenocarcinoma }\end{array} \\ 2018 & \end{array}$

He et al, Bladder urothelial 2018 carcinoma

Zhang Breast carcinoma et al, 2019

Lin et al, Esophageal 2018 adenocarcinoma

Lin et al, Stomach 2018 adenocarcinoma
30986203 AA-SHPRH-78032, AA-CASK-88861, AD-FBXL19-36205, AD-SAT2-39030, AD-TRO-89255, AD-CSTF2-89611, AP-ZC3H11 A-9456, AP-STK32C-13483, AP-GRB2-43439, AP-CRTC1-48500, AP-ERCC1-50440, AP-ESR1-78161, AT-MAGI3-4271, AT-TPM1-30982, AT-ATP8B3-46544, AT-MAST1-47878, AT-SPAG16-57327, AT-CBWD5-86498, AT-OLFM1-88103, ME-NSMF-193275, ME-GTF2H3-306194, RI-C11 orf49-15609, RI-ZNF276-38138, RI-USP36-43917, RI-NUDT18-82937, RI-NAPRT1-85430

30640723 AP-BDNF-14763, AP-DDX58-86057, AP-FYTTD1-68310, AP-GNAL-44643, AP-GPATCH2L-28538, AP-HUS1-79610, AP-MAP4-64545, AT-IPO11-72190, AT-ZFAND4-11368, ES-CKMT2-72660, ES-CMC2-37735, ES-FBXL12-47421, ES-NDUFB1-28987, ES-PSMD12-43112, ES-ZNF528-51457, RI-AP3M2-83565, RI-DNASE1L3-65424, RI-GABARAP-38871

30221674 ES-TCEB2-33303, AD-ABHD17A-46558, AP-FKBP2-16603, ES-TXN-87183, AP-FKBP2-16602, AD-YPEL3-36074, ES-STXBP2-47124, AT-PTGDS-88235, AT-HMGA2-22879, ES-NHLRC3-25701

30048970 AA-B4GALT2-1228, ES-TMTC2-9217, ES-TIMM9-11224, ES-APOBEC3D-26508, AP-TPD52-35921, ES-MICU1-4164, ES-DDX11-8115, ES-SMC6-22132

30984247 AA-CARM1-47598, AA-ZBTB25-27884, AA-GPBP1-72126, AA-ZNRF1-37578, AA-DDX41-74796, AA-CTDSP1-57478, AD-OS9-22701, AD-HN1-43371, AD-THTPA-26757, AD-NTMT1-87866, AD-MGME1-58753, AD-SEC31A-69735, AP-SEC22A-66462, AP-ALG3-67851, AP-PACS2-29630, AP-ECE2-67857, AP-HSP90AB1-76378, AT-MAGT1-89535, AT-RCBTB1-25898, AT-SIN3B-48214, AT-SARNP-22252, AT-ZNF675-48822, AT-STOX2-71289, AT-NIPAL3-1110, ES-NDUFA12-23737, ES-UBR4-880, ES-COPS3-39468, ES-ABCE1-70753, ES-CCNI-69628, ES-RPAP1-30096, ME-HLCS-96019, RI-RBM48-80441, RI-RBM6-64936, RI-RPAP1-30095, RI-METTL17-26476, RI-POMGNT1-2787, RI-TRABD-62792, RI-WDR6-64794, RI-FASTK-82335, RI-NAA38-81579

30131306 AA-U2AF1L4-49280, AA-TICRR-32428, AA-RSRC2-24968, AA-PREPL-53439, AA-PPIL2-61247, AA-FAM135A-76637, AA-CDV3-66839, AA-ABCB7-89517, AD-ZNF384-19927, AD-RPP14-65434, AD-PQBP1-89028, AD-MFSD11-43690, AD-COX6C-84682, AP-ZNF623-85469, AP-KIAA0513-37876, AP-FAM19A5-62732, AP-ALDH6A1-28367, AT-TRIM4-80864, AT-RNASEH2B-25927, AT-RNASEH2B-25926, AT-MCPH1-82574, AT-ARL6-65732, AT-AHI1-77886, ES-TNC-87345, ES-PML-31651, ES-NBPF15-91080, ES-MYL6-22384, ES-MRPL43-12857, ES-IRF9-117161, ME-SDR39U1-27012, ME-KLHL2-71038, ME-CMC2-37707, RI-ZNF131-71926, RI-SLC52A3-58464, RI-PPARGC1B-74051, RI-PCGF3-68404, RI-MDK-15570, RI-MAF-37687, RI-FAM9C-88504

30131306 AA-RPLP0-24727, AA-NAT6-64990, AA-MRVI1-14373, AA-LMO7-26065, AA-BDKRB2-29192, AD-YIPF2-47605, AD-SPHK2-50793, AD-SENP1-21411, AD-PGAP2-14004, AD-NFATC1-46241, AD-CCDC51-64653, AP-RCAN1-60494, AP-PLCD1-64009, AP-LTBP1-53179, AP-FAM65B-75537, AP-ABL2-9101, AT-ZNF846-47399, AT-ZFYVE28-68559, AT-STEAP4-80362, AT-STEAP4-80361, AT-KIF1B-602, AT-KIF1B-601, AT-CXCL12-11344, AT-CLDN11-67617, AT-ABCB5-78909, ES-UBXN11-1263, ES-TMEM230-58637, ES-SRSF3-75985, ES-SORBS1-12641, ES-P4HA2-73263, ES-CREM-11245, ME-N4BP2L1-25590, ME-KDM6A-98323, ME-FYN-77273, ME-CCDC53-106010, RI-TREX1-64682, RI-SRSF7-53276, RI-RPS15-46490, RI-LDHA-14642, RI-BICD2-86883, RI-ALS2CL-64462 
Table I. Continued.

\begin{tabular}{|c|c|c|c|c|}
\hline $\begin{array}{l}\text { Author, } \\
\text { year }\end{array}$ & Type & PMID & Alternative splicing events & (Refs.) \\
\hline $\begin{array}{l}\text { Lin et al, } \\
2018\end{array}$ & $\begin{array}{l}\text { Colon } \\
\text { adenocarcinoma }\end{array}$ & 30131306 & $\begin{array}{l}\text { AA-RASSF7-13691, AA-PTGR1-87219, AA-FAM173A-32964, } \\
\text { AA-DPP3-17040, AA-CDV3-66842, AD-RNF14-73855, } \\
\text { AD-IP6K2-64759, AD-HPS4-61506, AD-HDGF-8323, } \\
\text { AD-ANKRD46-84712, AD-ADPGK-31594, AP-TUBB3-38167, } \\
\text { AP-RAB3IP-23345, AP-MAZ-35938, AP-FADS2-16289, } \\
\text { AP-ENO2-20011, AT-ZNF765-51718, AT-UPK3B-80182, } \\
\text { AT-RASEF-86677, AT-RASEF-86676, AT-NRG4-31911, } \\
\text { AT-AIG1-77972, } \\
\text { ES-VTI1B-28083, ES-STRN3-27098, ES-RHOC-4236, } \\
\text { ES-PRMT1-51042, ES-PLEKHM2-767, ES-DMWD-50528, } \\
\text { ES-D2HGDH-58423, ME-CNOT10-63822, RI-ZNF226-50290, } \\
\text { RI-NPIPA5-34148, RI-ELP5-38889, RI-ALS2CL-64463 }\end{array}$ & (60) \\
\hline $\begin{array}{l}\text { Lin et al, } \\
2018\end{array}$ & $\begin{array}{l}\text { Rectal } \\
\text { adenocarcinoma }\end{array}$ & 30131306 & $\begin{array}{l}\text { AA-ZNF467-82205, AA-RNPC3-3907, AA-GGT1-61440, } \\
\text { AA-BTN3A1-75660, AD-OSBPL9-2975, AD-METTL23-43637, } \\
\text { AD-BCS1L-57522, AP-TADA2B-68732, AP-PTCH1-86955, } \\
\text { AP-DAB2IP-87442, AT-PUS10-53676, AT-NOTCH2NL-4437, } \\
\text { ES-SPAG9-42496, ES-SERPINA1-29134, ES-PHB2-20048, } \\
\text { ES-FGFR1OP2-20856, ME-RBMS2-22465, RI-ZNF692-10557, } \\
\text { RI-WDR33-55246, RI-TMEM91-50046, RI-SIDT2-18886, } \\
\text { RI-EXOSC9-70501, RI-ADARB1-60863 }\end{array}$ & (60) \\
\hline
\end{tabular}

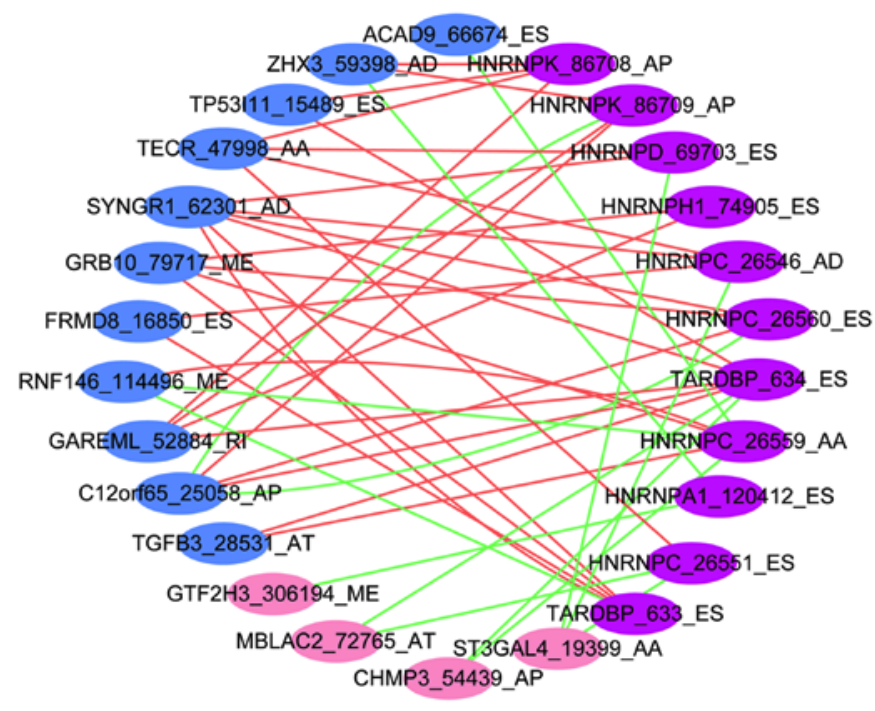

Figure 8. Associations between overall survival-related alternative splicing events and splicing factors. Each purple point represents an alternative splicing event of a single risk factor and a significant overall survival-related splicing factor $(\mathrm{P}<0.05)$. Each blue point represents a positive overall survival-related alternative splicing event identified by multivariate Cox analysis $(\mathrm{P}<0.001)$. Each pink point represents a negative overall survival-related alternative splicing event identified by multivariate Cox analysis $(\mathrm{P}<0.001)$. Red lines represent positive correlations $(r>0.3 ; \mathrm{P}<0.05)$; green lines represent negative correlations $(r<-0.3$; $\mathrm{P}<0.05)$.

these genes remains controversial and has not been applied clinically (72). Due to the complexity of cancer, diagnosis or prognosis based on a single molecule is limited. The advent of high-throughput sequencing technologies allows us to address this issue by identifying more genomic abnormalities related to CCA.
Using the TCGASpliceSeq CCA data, 38,804 AS events were identified in 9,673 CCA genes, some of which exhibited multiple AS events occurring simultaneously. AS events are widespread in CCA and may be related to its occurrence and development. For instance, the first AS event reported to be associated with CCA prognosis was exon 2 skipping of the trefoil factor 2 (TFF2) gene (80). TFF2 is highly expressed in a variety of tumors, including CCA. Exon 2 skipping AS events lead to the loss of exon 2, resulting in a decrease in wild-type TFF2 proteins. A high proportion of this AS event in TFF2 is associated with improved prognosis. Another example is P53, as alterations in the $\mathrm{N}$-terminus of this protein by AS has been demonstrated to worsen the prognosis in patients with CCA (81).

The present study identified a large number of CCA AS events, 1,639 of which were correlated with patient prognosis $(\mathrm{P}<0.05)$. Bioinformatics analyses of all genes with significant prognostic values identified the following genes to be located at the center of the gene network: MAX, MAPK11, GABARAPL1, CHEK1, XIAP and FOXA1. These genes have been broadly reported to be involved in cell cycle regulation, autophagy, proliferation, apoptosis and have been previously demonstrated to be associated with cancer (82-87). Tumor-specific AS events have been intensively studied by investigating the differentially alternatively spliced genes between tumor and adjacent normal tissues (88) in certain cancer types, such as breast (89), lung (56) and ovarian (57) cancer. Yosudjai et al (90) also demonstrated that aberrant AS of anterior gradient protein 2 homolog promoted cell proliferation, migration, invasion and adhesive potential in CCA; however, there has been a lack of research on its association with patient prognoses. Among the genes identified above, $M A X$ forms a heterodimer with the proto-oncogene $M Y C$, which binds to DNA to regulate the transcription of multiple genes, thus regulating cell 
Table II. Prediction models for cholangiocarcinoma based on each type of splicing event.

\begin{tabular}{|c|c|c|c|c|}
\hline Risk score & Algorithm & $\mathrm{HR}(95 \% \mathrm{CI})$ & P-value & AUC \\
\hline Risk score (AA) & $\begin{array}{l}\text { PSI }_{\text {ST3GAL4_19399_AA }} \text { x } 0.849+ \\
\text { PSI }_{\text {TECR_47998_AA }} \times(-6.242)+ \\
\text { PSI }_{\text {TGIF1_44506_AA }} \times(-0.713)\end{array}$ & $10.990(3.818-31.61)$ & $<0.001$ & 0.901 \\
\hline Risk score (AD) & $\begin{array}{l}\text { PSI }_{\text {SYNGR1_62301_AD }} \text { X (-0.645) + } \\
\text { PSI }_{\text {ZHX3_59398_AD }} \text { X (-0.277) }\end{array}$ & $3.375(1.227-9.283)$ & 0.018 & 0.858 \\
\hline Risk score (AP) & $\begin{array}{l}\text { PSI }_{\text {C12orf65_25058_AP }} \text { X }(-0.147)+ \\
\text { PSI }_{\text {CHMP3_54439_AP }} \text { X } 1.274+ \\
\text { PSI }_{\text {SH3KBP1_88640_AP }} \text { X } 0.432\end{array}$ & $17.910(5.658-56.67)$ & $<0.001$ & 0.986 \\
\hline Risk score (AT) & $\begin{array}{l}\mathrm{PSI}_{\text {MBLAC2_72765_AT }} \text { x } 0.166+ \\
\text { PSI }_{\text {TGFB3_28531_AT }} \text { X }(-1.244)\end{array}$ & $7.622(2.576-22.55)$ & $<0.001$ & 0.839 \\
\hline Risk score (ES) & $\begin{array}{l}\text { PSI }_{\text {ACAD9_66674_ES }} \text { x }(-1.952)+ \\
\text { PSI }_{\text {FRMD8_16850_ES }} \text { x }(-0.701)+ \\
\text { PSI }_{\text {PLEKHG2_49826_ES }} \text { X }(-1.49)+ \\
\text { PSI }_{\text {TP53111_15489_ES }} \text { x } 2.014+ \\
\text { PSI }_{\text {UBE2F_58170_ES }} \text { X } 8.384\end{array}$ & $19.090(5.705-63.86)$ & $<0.001$ & 0.939 \\
\hline Risk score (ME) & $\begin{array}{l}\text { PSI }_{\text {FGFR3_68513_ME }} \text { X }(-0.15)+ \\
\text { PSI }_{\text {GRB10_79717_ME }} \text { X }(-0.068)+ \\
\text { PSI }_{\text {GTF2H3_306194_ME }} \text { X } 1.421+ \\
\text { PSI }_{\text {RNF146_114496_ME }} \times(-0.085)+ \\
\text { PSI } \\
\text { SORBS2_71377_ME } \text { (-0.282) }\end{array}$ & $4.977(1.790-13.84)$ & 0.002 & 0.779 \\
\hline Risk score (RI) & $\begin{array}{l}\mathrm{PSI}_{\text {C11orf88_18667_RI }} \times(-0.073)+ \\
\text { PSI }_{\text {DET1_32385_RI }} \times(-0.214)+ \\
\text { PSI }_{\text {GAREML_52884_RI }} \times(-0.332)\end{array}$ & $6.358(2.295-17.61)$ & $<0.001$ & 0.859 \\
\hline Risk score (merged) & $\begin{array}{l}\text { PSI }_{\text {TFDP1_26387_AD }} \times(-0.57)+ \\
\text { PSI }_{\text {MBLAC2_72766_AT }} \text { x }(-0.838)+ \\
\text { PSI }_{\text {HACL1_63592_ES }} \times(-0.62)+ \\
\text { PSI }_{\text {PLEKHG2_49826_ES }} \text { x }(-4.314)+ \\
\text { PSI }_{\text {UBE2F_58170_ES }} \text { X } 7.06\end{array}$ & $19.670(5.842-66.22)$ & $<0.001$ & 0.984 \\
\hline
\end{tabular}

PSI, percent spliced index; HR, Hazard ratio; CI, confidence interval; AUC, area under the curve; AA, alternate acceptor; AD, alternate donor; AP, alternate promoter; AT, alternate terminator; ES, exon skip; ME, mutually exclusive exon; RI, retained intron; 'ST3GAL4_19399_AA' represents 'gene symbol_alternative splicing event ID_splice type'.

proliferation, differentiation and apoptosis (82). The MAPK11 gene encodes a serine/threonine kinase that is widely involved in various cellular signal transduction pathways, such as p38 the MAPK signaling pathway by phosphorylating multiple target proteins (87). $C H E K 1$ is a cell cycle monitoring-related protein, abnormalities of which result in DNA damage and the bypassing of the cell cycle checkpoints (91). FOXAl is a transcription factor, the aberrant expression of which is closely associated with hepatocellular carcinoma (84); however, its association with CCA has not been previously reported.

The current study is the first to identify the characteristics of alternative CCA splicing events and establish a model that can predict CCA prognosis using PIs from AS events. Li et al (56), who studied non-small cell lung cancer, were the first to demonstrate a combined survival and correlation network between the expression of splicing factors and AS events; the present study provides a more accurate potential network by screening the significant prognostic AS events identified by univariate Cox regression.
The present study attempted to predict the prognosis of patients with CCA using the three most significant AS events and genes. The results were not satisfactory (the maximum AUC was 0.747; data not shown), likely due to individual differences between patients. Similarly, no single factor was able to accurately predict patient prognosis. Therefore, ten AS events with the most significant prognostic values, as calculated by multivariate Cox regression analysis for each type of AS event, were selected to construct a PI. The prognostic models based on the combined seven types of splicing events demonstrated comparable predictive efficacy to the model that used only the AP splicing events. However, the combined model was more successful at predicting patient prognosis compared with models based on any other single type of splicing event.

The results of the present study were compared with those reported in previous studies. All AS events identified in the present study were uniquely associated with CCA, with the exception of ME-GTF2H3-306194, which has been previously identified to have prognostic value in papillary thyroid cancer 
Table III. Predictive factors identified for cholangiocarcinoma using multivariate logistic regression.

\begin{tabular}{|c|c|c|}
\hline Splicing type & Gene symbol & AS ID \\
\hline $\mathrm{AA}$ & ST3GAL4 & 19399 \\
\hline $\mathrm{AA}$ & TECR & 47998 \\
\hline $\mathrm{AA}$ & TGIF1 & 44506 \\
\hline $\mathrm{AD}$ & SYNGR1 & 62301 \\
\hline $\mathrm{AD}$ & ZHX3 & 59398 \\
\hline $\mathrm{AP}$ & C12orf65 & 25058 \\
\hline AP & CHMP3 & 54439 \\
\hline AP & SH3KBP1 & 88640 \\
\hline AT & MBLAC2 & 72765 \\
\hline AT & TGFB3 & 28531 \\
\hline $\mathrm{ES}$ & PLEKHG2 & 49826 \\
\hline $\mathrm{ES}$ & ACAD9 & 66674 \\
\hline $\mathrm{ES}$ & TP53I11 & 15489 \\
\hline $\mathrm{ES}$ & FRMD8 & 16850 \\
\hline ES & UBE2F & 58170 \\
\hline $\mathrm{ME}$ & FGFR3 & 68513 \\
\hline $\mathrm{ME}$ & RNF146 & 114496 \\
\hline $\mathrm{ME}$ & GRB10 & 79717 \\
\hline $\mathrm{ME}$ & SORBS2 & 71377 \\
\hline $\mathrm{ME}$ & GTF2H3 & 306194 \\
\hline RI & C11orf88 & 18667 \\
\hline RI & DET1 & 32385 \\
\hline RI & GAREML & 52884 \\
\hline
\end{tabular}

$\mathrm{AA}$, alternate acceptor; $\mathrm{AD}$, alternate donor; $\mathrm{AP}$, alternate promoter; AT, alternate terminator; ES, exon skip; ME, mutually exclusive exon; RI, retained intron; AS ID, alternative splicing event ID.

and CCA, indicating that ME-GTF2H3-306194 may serve similar functions in CCA and papillary thyroid cancer and suggesting a new treatment possibility for CCA and papillary thyroid cancer by targeting ME-GTF2H3-306194.

In addition, the existence of two AS events predicted by the TCGASpliceSeq database was verified using PCR analysis of six CCA tumor tissue samples. Metastasis was common in cholangiocarcinoma $(7,92)$. The direct infiltration of cholangiocarcinoma cells along the bile duct wall is one of the main features of cholangiocarcinoma metastasis (7). Cancer cells diffuse invasively in the bile duct wall and coexist with the bile duct and surrounding connective tissue, making the infiltration range of cholangiocarcinoma difficult to identify, and thus non-cancerous bile duct tissues cannon be easily obtained during surgery (93). Therefore, only two AS events were verified, and the difference of the splicing events between tumor and non-tumor tissues was not determined.

To investigate the causes of AS events in CCA, the relationship between AS events and splicing factors was analyzed. Splicing factors are a class of RNA-binding proteins that affect the selection of cleavage sites by recognizing the cis-acting elements of mRNA precursors (27). Previous studies on lung (56), ovarian (57) and bladder (94) cancer have demonstrated that the expression of splicing factors was correlated with prognosis-related AS events; however, this phenomenon was not observed in the current study. Pearson's correlation analysis between a group of splicing factors, the expression of which was directly related to AS events, and general OS-related splicing events identified 15 AS events that were mutually associated. Therefore, in CCA, splicing factors may affect the structure of their own proteins by creating their own AS event and then subsequently affecting the AS events of other genes.

In summary, TCGASpliceSeq data were mined to identify the characteristics of AS events associated with CCA to establish a model that can predict CCA prognosis using PIs from AS events. The prognostic effect was estimated using ROC curves and was favorable, with AUCs $>0.9$. However, due to the small sample size, the validity of this model should be verified with additional clinical samples and in different populations. Several additional limitations of the present study also need to be addressed. The data in the current study were obtained from online databases and thus remain at the bioinformatics level. Additionally, among the AS events selected to construct the PI model, one event was not CCA-specific, which reduced the specificity of the PI model. In addition, although splicing factors have been demonstrated to contribute to changes in the splicing patterns of target genes and tumorigenesis, and certain cancer-specific splicing factors are associated with OS rates, there has been a lack of research on CCA-related splicing factors, and whether the splicing factors identified in the present study are truly CCA-specific requires additional research. Finally, although an accurate PI model was constructed by screening the significant prognostic AS events identified by univariate and multivariate Cox regression, the results are based on a single cohort and should be validated in additional cohorts; this is intended to be performed in our subsequent study.

\section{Acknowledgements}

Not applicable.

\section{Funding}

The study was supported by the Promoting Project of Basic Capacity for Young and Middle-aged University Teachers in Guangxi (grant no. 2017KY0111), the Innovation Project of Guangxi Graduate Education (grant no. YCBZ2017045), the National Natural Science Foundation of China (grant nos. 31760319, 81360066 and 81660241) and the Guangxi First-class Discipline Project for Pharmaceutical Sciences (grant no. GXFCDP-PS-2018).

\section{Availability of data and materials}

The datasets generated and analyzed during the current study are available from the TCGA data portal (portal.gdc. cancer.gov/).

\section{Authors' contributions}

HYW analyzed and interpreted data, and drafted the manuscript. YW, LML, ZBC, QPH and SLP obtained information from the database, supervised the data mining, and revised 
the manuscript. All authors read and approved the final manuscript.

\section{Ethics approval and consent to participate}

The study was approved by the Ethics Committee of the First Affiliated Hospital of Guangxi Medical University (ethics approval no. 20170303-2). All the patients provided written informed consent to participate in this study.

\section{Patient consent for publication}

Not applicable.

\section{Competing interests}

The authors declare that they have no competing interests.

\section{References}

1. Popat K, McQueen K and Feeley TW: The global burden of cancer. Best Pract Res Clin Anaesthesiol 27: 399-408, 2013.

2. Verathamjamras C, Weeraphan C, Chokchaichamnankit D, Watcharatanyatip K, Subhasitanont P, Diskul-Na-Ayudthaya P, Mingkwan K, Luevisadpaibul V, Chutipongtanate S, Champattanachai $\mathrm{V}$, et al: Secretomic profiling of cells from hollow fiber bioreactor reveals PSMA3 as a potential cholangiocarcinoma biomarker. Int J Oncol 51: 269-280, 2017.

3. Lu X, Zhou C, Li R, Deng Y, Zhao L and Zhai W: Long noncoding RNA AFAP1-AS1 promoted tumor growth and invasion in cholangiocarcinoma. Cell Physiol Biochem 42: 222-230, 2017.

4. Cai X, Li J, Yuan X, Xiao J, Dooley S, Wan X, Weng H and Lu L: CD133 expression in cancer cells predicts poor prognosis of non-mucin producing intrahepatic cholangiocarcinoma. J Transl Med 16: 50, 2018.

5. Luo G, Li B, Duan C, Cheng Y, Xiao B, Yao F, Wei M, Tao Q, Feng C, Xia X, et al: cMyc promotes cholangiocarcinoma cells to overcome contact inhibition via the mTOR pathway. Oncol Rep 38: 2498-2506, 2017.

6. Qian Y, Yao W, Yang T, Yang Y, Liu Y, Shen Q, Zhang J, Qi W and Wang J: aPKC-iota/P-Sp1/Snail signaling induces epithelial-mesenchymal transition and immunosuppression in cholangiocarcinoma. Hepatology 66: 1165-1182, 2017.

7. Razumilava N and Gores GJ: Cholangiocarcinoma. Lancet 383 : 2168-2179, 2014.

8. Zhou G, Yang Z, Wang X, Tao R and Zhou Y: TRAIL enhances shikonin induced apoptosis through ROS/JNK signaling in cholangiocarcinoma cells. Cell Physiol Biochem 42: 1073-1086, 2017

9. Tang J, Liao Y, He S, Shi J, Peng L, Xu X, Xie F, Diao N, Huang J, Xie $\mathrm{Q}$, et al: Autocrine parathyroid hormone-like hormone promotes intrahepatic cholangiocarcinoma cell proliferation via increased ERK/JNK-ATF2-cyclin D1 signaling. J Transl Med 15: 238, 2017.

10. Chng KR, Chan SH, Ng AHQ, Li C, Jusakul A, Bertrand D, Wilm A, Choo SP, Tan DMY, Lim KH, et al: Tissue microbiome profiling identifies an enrichment of specific enteric bacteria in opisthorchis viverrini associated cholangiocarcinoma. Ebiomedicine 8: 195-202, 2016.

11. Breitbart RE, Andreadis A and Nadalginard B: Alternative splicing: A ubiquitous mechanism for the generation of multiple protein isoforms from single genes. Annu Rev Biochem 56: 467-495, 1987.

12. Blencowe BJ: Alternative splicing: New insights from global analyses. Cell 126: 37-47, 2006.

13. Oltean S and Bates DO: Hallmarks of alternative splicing in cancer. Oncogene 33: 5311-5318, 2013.

14. Wang ET, Rickard S, Luo S, Khrebtukova I, Zhang L, Mayr C, Kingsmore SF, Schroth GP and Burge CB: Alternative isoform regulation in human tissue transcriptomes. Nature 456: 470-476, 2008.

15. Pan Q, Shai O, Lee LJ, Frey BJ and Blencowe BJ: Deep surveying of alternative splicing complexity in the human transcriptome by high-throughput sequencing. Nat Genet 40: 1413-1415, 2008.
16. Dvinge $\mathrm{H}$ and Bradley RK: Widespread intron retention diversifies most cancer transcriptomes. Genome Medi 7: 45, 2015.

17. Bechara EG, Sebestyén E, Bernardis I, Eyras E and Valcárcel J: RBM5, 6 and 10 differentially regulate NUMB alternative splicing to control cancer cell proliferation. Mol Cell 52: 720-733, 2013

18. Ghigna C, Giordano S, Shen H, Benvenuto F, Castiglioni F, Comoglio PM, Green MR, Riva S and Biamonti G: Cell motility is controlled by SF2/ASF through alternative splicing of the Ron Protooncogene. Mol Cell 20: 881-890, 2005.

19. Jung H, Lee D, Lee J, Park D, Kim YJ, Park WY, Hong D, Park PJ and Lee E: Intron retention is a widespread mechanism of tumor-suppressor inactivation. Nat Genet 47: 1242-1248, 2015.

20. Goldstein L, Lee J, Gnad F, Klijn C, Schaub A, Reeder J, Daemen A, Bakalarski CE, Holcomb T, Shames DS, et al: Recurrent loss of NFE2L2 exon 2 is a mechanism for Nrf2 pathway activation in human cancers. Cell Rep 16: 2605-2617, 2016.

21. Kornblihtt AR: Epigenetics at the base of alternative splicing changes that promote colorectal cancer. J Clin Invest 127: 3281-3283, 2017.

22. Poulikakos PI, Persaud Y, Janakiraman M, Kong X, Ng C, Moriceau G, Shi H, Atefi M, Titz B, Gabay MT, et al: RAF inhibitor resistance is mediated by dimerization of aberrantly spliced BRAF (V600E). Nature 480: 387-390, 2011.

23. Sotillo E, Barrett DM, Black KL, Bagashev A, Oldridge D, Wu G, Sussman R, Lanauze C, Ruella M, Gazzara MR, et al: Convergence of acquired mutations and alternative splicing of CD19 enables resistance to CART-19 immunotherapy. Cancer Discov 5: 1282-1295, 2015.

24. Wang BD, Ceniccola K, Hwang S, Andrawis R, Horvath A, Freedman JA, Olender J, Knapp S, Ching T, Garmire L, et al: Alternative splicing promotes tumour aggressiveness and drug resistance in African American prostate cancer. Nat Commun 8: 15921, 2017.

25. Sebestyén E, Zawisza M and Eyras E: Detection of recurrent alternative splicing switches in tumor samples reveals novel signatures of cancer. Nucleic Acids Res 43: 1345-1356, 2015.

26. Trincado JL, Sebestyén E, Pagés A and Eyras E: The prognostic potential of alternative transcript isoforms across human tumors. Genome Med 8: 85, 2016.

27. Shen S, Wang Y, Wang C, Ying NW and Yi X: SURVIV for survival analysis of mRNA isoform variation. Nat Commun 7: 11548, 2016.

28. Parsons DW, Li M, Zhang X, Jones S, Leary RJ, Lin JC, Boca SM, Carter H, Samayoa J, Bettegowda C, et al: The genetic landscape of the childhood cancer medulloblastoma. Science 331: 435-439, 2011.

29. Ghigna C, De Toledo M, Bonomi S, Valacca C, Gallo S, Apicella M, Eperon I, Tazi J and Biamonti G: Pro-metastatic splicing of Ron proto-oncogene mRNA can be reversed: Therapeutic potential of bifunctional oligonucleotides and indole derivatives. RNA Biol 7: 495-503, 2010.

30. Lee CW and Abdelwahab O: Therapeutic targeting of splicing in cancer. Nat Med 22: 976-986, 2016.

31. Koh CM, Bezzi M, Low DH, Ang WX, Teo SX, Gay FP, Al-Haddawi M, Tan SY, Osato M, Sabò A, et al: MYC regulates the core pre-mRNA splicing machinery as an essential step in lymphomagenesis. Nature 523: 96-100, 2015.

32. Havens MA and Hastings ML: Splice-switching antisense oligonucleotides as therapeutic drugs. Nucleic Acids Res 44: 6549-6563, 2016

33. Salton M and Misteli T: Small molecule modulators of Pre-mRNA splicing in cancer therapy. Trends Mol Med 22: 28-37, 2016.

34. Gao L, Xie ZC, Pang JS, Li TT and Chen G: A novel alternative splicing-based prediction model for uteri corpus endometrial carcinoma. Aging (Albany NY) 11: 263-283, 2019.

35. Xiong Y, Deng Y, Wang K, Zhou H, Zheng X, Si L and Fu Z: Profiles of alternative splicing in colorectal cancer and their clinical significance: A study based on large-scale sequencing data. EBioMedicine 36: 183-195, 2018.

36. Huang ZG, He RQ and Mo ZN: Prognostic value and potential function of splicing events in prostate adenocarcinoma. Int J Oncol 53: 2473-2487, 2018.

37. Jusakul A, Cutcutache I, Yong CH, Lim JQ, Huang MN, Padmanabhan N, Nellore V, Kongpetch S, Ng AWT, Ng LM, et al: Whole-genome and epigenomic landscapes of etiologically distinct subtypes of cholangiocarcinoma. Cancer Discov 7: 1116-1135, 2017.

38. Supek F, Miñana B, Valcárcel J, Gabaldón T and Lehner B: Synonymous mutations frequently act as driver mutations in human cancers. Cell 156: 1324-1335, 2014. 
39. Sterneweiler $\mathrm{T}$ and Sanford JR: Exon identity crisis: Disease-causing mutations that disrupt the splicing code. Genome Biol 15: 201, 2014.

40. Diederichs S, Bartsch L, Berkmann JC, Fröse K, Heitmann J, Hoppe C, Iggena D, Jazmati D, Karschnia P, Linsenmeier M, et al: The dark matter of the cancer genome: Aberrations in regulatory elements, untranslated regions, splice sites, non-coding RNA and synonymous mutations. EMBO Mol Med 8: 442-457, 2016.

41. Singh S, Narayanan SP, Biswas K, Gupta A, Ahuja N, Yadav S, Panday RK, Samaiya A, Sharan SK and Shukla S: Intragenic DNA methylation and BORIS-mediated cancer-specific splicing contribute to the Warburg effect. Proc Natl Acad Sci USA 114 11440-11445, 2017.

42. Gelfman S, Cohen N, Yearim A and Ast G: DNA-methylation effect on cotranscriptional splicing is dependent on GC architecture of the exon-intron structure. Genome Res 23: 789-799, 2013

43. Shukla S, Kavak E, Gregory M, Imashimizu M, Shutinoski B, Kashlev M, Oberdoerffer P, Sandberg R and Oberdoerffer S: CTCF-promoted RNA polymerase II pausing links DNA methylation to splicing. Nature 479: 74-79, 2011.

44. Yuan H, Li N, Fu D, Ren J, Hui J, Peng J, Liu Y, Qiu T, Jiang M, Pan $\mathrm{Q}$, et al: Histone methyltransferase SETD2 modulates alternative splicing to inhibit intestinal tumorigenesis. J Clin Invest 127: 3375-3391, 2017.

45. Ding X, Liu S, Tian M, Zhang W, Zhu T, Li D, Wu J, Deng H, Jia Y, Xie W, et al: Activity-induced histone modifications govern Neurexin-1 mRNA splicing and memory preservation. Nat Neurosci 20: 690-699, 2017.

46. Sharma A, Nguyen H, Geng C, Hinman MN, Luo G and Lou $H$ : Calcium-mediated histone modifications regulate alternative splicing in cardiomyocytes. Proc Natl Acad Sci USA 111: E4920-E4928, 2014.

47. Kim S, Kim H, Fong N, Erickson B and Bentley DL: Pre-mRNA splicing is a determinant of histone H3K36 methylation. Proc Natl Acad Sci USA 108: 13564-13569, 2011.

48. Takehara T, Liu X, Fujimoto J, Friedman SL and Takahashi H: Expression and role of Bcl-xL in human hepatocellular carcinomas. Hepatology 34: 55-61, 2001.

49. Boise LH, Gonzálezgarcía M, Postema CE, Ding L, Lindsten T, Turka LA, Mao X, Nuñez G and Thompson CB: bcl-x, a bcl-2-related gene that functions as a dominant regulator of apoptotic cell death. Cell 74: 597-608, 1993.

50. Bingle CD, Craig RW, Swales BM, Singleton V, Zhou P and Whyte MK: Exon skipping in Mcl-1 results in a Bcl-2 homology domain 3 only gene product that promotes cell death. J Biol Chem 275: 22136-22146, 2000.

51. Bae J, Leo CP, Hsu SY and Hsueh AJ: MCL-1S, a splicing variant of the antiapoptotic BCL-2 family member MCL-1, encodes a proapoptotic protein possessing only the $\mathrm{BH} 3$ domain. J Biol Chem 275: 25255-25261, 2000.

52. Salton M, Kasprzak WK, Voss T, Shapiro BA, Poulikakos PI and Misteli T: Inhibition of vemurafenib-resistant melanoma by interference with pre-mRNA splicing. Nat Commun 6: 7103, 2015.

53. Chen K, Xiao H, Zeng J, Yu G, Zhou H, Huang C, Yao W, Xiao W, Hu J, Guan W, et al: Alternative splicing of EZH2 pre-mRNA by SF3B3 contributes to the tumorigenic potential of renal cancer. Clin Cancer Res 23: 3428-3441, 2017.

54. Luo C, Cheng Y, Liu Y, Chen L, Liu L, Wei N, Xie Z, Wu W and Feng Y: SRSF2 regulates alternative splicing to drive hepatocellular carcinoma development. Cancer Res 77: 1168-1178, 2017.

55. Calabretta S, Bielli P, Passacantilli I, Pilozzi E, Fendrich V, Capurso G, Fave GD and Sette C: Modulation of PKM alternative splicing by PTBP1 promotes gemcitabine resistance in pancreatic cancer cells. Oncogene 35: 2031-2039, 2016.

56. Li Y, Sun N, Lu Z, Sun S, Huang J, Chen Z and He J: Prognostic alternative mRNA splicing signature in non-small cell lung cancer. Cancer Lett 393: 40-51, 2017.

57. Zhu J, Chen Z and Yong L: Systematic profiling of alternative splicing signature reveals prognostic predictor for ovarian cancer. Gynecol Oncol 148: 368-374, 2018.

58. Miles S, Swift L and Leinster SJ: The dundee ready education environment measure (DREEM): A review of its adoption and use. Med Teach 34: e620-e634, 2012.

59. Shi Y,ChenZ, GaoJ,Wu S, GaoH and Feng G: Transcriptome-wide analysis of alternative mRNA splicing signature in the diagnosis and prognosis of stomach adenocarcinoma. Oncol Rep 40 2014-2022, 2018.
60. Lin P, He RQ, Ma FC, Liang L, He Y, Yang H, Dang YW and Chen G: Systematic analysis of survival-associated alternative splicing signatures in gastrointestinal pan-adenocarcinomas. EBioMedicine 34: 46-60, 2018.

61. Ryan M, Wong WC, Brown R, Akbani R, Su X, Broom B, Melott J and Weinstein J: TCGASpliceSeq a compendium of alternative mRNA splicing in cancer. Nucleic Acids Res 44: D1018-D1022, 2016.

62. R Core Team. R: A language and environment for statistical computing. R Foundation for Statistical Computing, Vienna, Austria, 2012. ISBN 3-900051-07-0, URL http://www.R-project. org/.

63. Smidt N DJaMT: Guide to the contents of a Cochrane review and protocol. Cochrane handbook for systematic reviews of diagnostic test accuracy, 2011.

64. Conway JR, Lex A and Gehlenborg N: UpSetR: An R package for the visualization of intersecting sets and their properties. Bioinformatics 33: 2938-2940, 2017.

65. Wu G, Dawson E, Duong A, Haw R and Stein L: ReactomeFIViz: A Cytoscape app for pathway and network-based data analysis. F1000Res 3: 146, 2014.

66. Shannon P, Markiel A, Ozier O, Baliga NS, Wang JT, Ramage D, Amin N, Schwikowski B and Ideker T: Cytoscape: A software environment for integrated models of biomolecular interaction networks. Genome Res 13: 2498-2504, 2003.

67. Wickham H: ggplot2: Elegant Graphics for Data Analysis. Springer-Verlag, New York, NY, pp 77-186, 2016.

68. Heagerty PJ and Zheng Y: Survival model predictive accuracy and ROC curves. Biometrics 61: 92-105, 2005.

69. Lee Y and Rio DC: Mechanisms and regulation of alternative Pre-mRNA splicing. Annu Rev Biochem 84: 291-323, 2015.

70. Piva F, Giulietti M, Burini AB and Principato G: SpliceAid 2: A database of human splicing factors expression data and RNA target motifs. Hum Mutat 33: 81-85, 2012.

71. Rahnemai-Azar AA, Weisbrod A, Dillhoff M, Schmidt C and Pawlik TM: Intrahepatic cholangiocarcinoma: Molecular markers for diagnosis and prognosis. Surg Oncol 26: 125-137, 2017.

72. Khan SA, Davidson BR, Goldin RD, Heaton N, Karani J, Pereira SP, Rosenberg WM, Tait P, Taylor-Robinson SD, Thillainayagam AV, et al: Guidelines for the diagnosis and treatment of cholangiocarcinoma: An update. Gut 61: 1657-1669, 2012.

73. Pasquali C, Sperti C, D'Andrea AA, Costantino V, Filipponi C and Pedrazzoli S: CA50 as a serum marker for pancreatic carcinoma: Comparison with CA19-9. Eur J Cancer 30A: 1042-1043, 1994.

74. Shan M, Tian Q and Zhang L: Serum CA50 levels in patients with cancers and other diseases. Prog Mol Biol Transl Sci 162: 187-198, 2019.

75. Dou H, Sun G and Zhang L: CA242 as a biomarker for pancreatic cancer and other diseases. Prog Mol Biol Transl Sci 162: 229-239, 2019.

76. Huang CK, Aihara A, Iwagami Y, Yu T, Carlson R, Koga H, Kim M, Zou J, Casulli S and Wands JR: Expression of transforming growth factor $\beta 1$ promotes cholangiocarcinoma development and progression. Cancer Lett 380: 153-162, 2016.

77. Churi CR, Shroff R, Wang Y, Rashid A, Kang HC, Weatherly J, Zuo M, Zinner R, Hong D, Meric-Bernstam F, et al: Mutation profiling in cholangiocarcinoma: Prognostic and therapeutic implications. PLoS One 9: e115383, 2014.

78. Dana P, Kariya R, Vaeteewoottacharn K, Sawanyawisuth K, Seubwai W, Matsuda K, Okada S and Wongkham S: Upregulation of CD147 promotes metastasis of cholangiocarcinoma by modulating the Epithelial-to-Mesenchymal transitional process. Oncol Res 25: 1047-1059, 2017.

79. Schweitzer N and Vogel A: Systemic therapy of cholangiocarcinoma: From chemotherapy to targeted therapies. Best Pract Res Clin Gastroenterol 29: 345-353, 2015.

80. Kamlua S, Patrakitkomjorn S, Jearanaikoon P, Menheniott TR, Giraud AS and Limpaiboon T: A novel TFF2 splice variant ( $\triangle \mathrm{EX} 2 \mathrm{TFF} 2)$ correlates with longer overall survival time in cholangiocarcinoma. Oncol Rep 27: 1207-1212, 2012.

81. Nutthasirikul N, Limpaiboon T, Leelayuwat C, Patrakitkomjorn $S$ and Jearanaikoon P: Ratio disruption of the $\Delta 133 \mathrm{p} 53$ and TAp53 isoform equilibrium correlates with poor clinical outcome in intrahepatic cholangiocarcinoma. Int J Oncol 42: 1181-1188, 2013.

82. Comino-Mendez I, Leandro-Garcia LJ, Montoya G, Inglada-Pérez L, de Cubas AA, Currás-Freixes M, Tysoe C, Izatt L, Letón R, Gómez-Graña Á, et al: Functional and in silico assessment of MAX variants of unknown significance. J Mol Med (Berl) 93: 1247-1255, 2015. 
83. Huang X, Wu Z, Mei Y and Wu M: XIAP inhibits autophagy via XIAP-Mdm2-p53 signalling. EMBO J 32: 2204-2216, 2013.

84. Li Z, Tuteja G, Schug J and Kaestner KH: Foxa1 and Foxa2 are essential for sexual dimorphism in liver cancer. Cell 148: 72-83, 2012.

85. McNeely S, Beckmann R and Bence Lin AK: CHEK again: Revisiting the development of CHK1 inhibitors for cancer therapy. Pharmacol Ther 142: 1-10, 2014.

86. Mo J, Zhang D and Yang R: MicroRNA-195 regulates proliferation, migration, angiogenesis and autophagy of endothelial progenitor cells by targeting GABARAPL1. Biosci Rep 36: pii: e00396, 2016.

87. Wagner EF and Nebreda AR: Signal integration by JNK and p38 MAPK pathways in cancer development. Nat Rev Cancer 9: 537-549, 2009.

88. Eswaran J, Horvath A, Godbole S, Reddy SD, Mudvari P, Ohshiro K, Cyanam D, Nair S, Fuqua SA, Polyak K, et al: RNA sequencing of cancer reveals novel splicing alterations. Sci Rep 3: 1689, 2013.

89. Suo C, Hrydziuszko O, Lee D, Pramana S, Saputra D, Joshi H, Calza S and Pawitan Y: Integration of somatic mutation, expression and functional data reveals potential driver genes predictive of breast cancer survival. Bioinformatics 31: 2607-2613, 2015.

90. Yosudjai J, Inpad C, Chomwong S, Dana P, Sawanyawisuth K, Phimsen S, Wongkham S, Jirawatnotai S and Kaewkong W: An aberrantly spliced isoform of anterior gradient-2, AGR2vH promotes migration and invasion of cholangiocarcinoma cell. Biomed Pharmacother 107: 109-116, 2018.

91. Mcneely S, Beckmann R and Bence Lin AK: CHEK again: Revisiting the development of CHK1 inhibitors for cancer therapy. Pharmacol Ther 142: 1-10, 2014.
92. Zhang H, Yang T, Wu M and Shen F: Intrahepatic cholangiocarcinoma: Epidemiology, risk factors, diagnosis and surgical management. Cancer Lett 379: 198-205, 2016.

93. Goere D, Wagholikar GD, Pessaux P, Carrère N, Sibert A, Vilgrain V, Sauvanet A and Belghiti J: Utility of staging laparoscopy in subsets of biliary cancers: Laparoscopy is a powerful diagnostic tool in patients with intrahepatic and gallbladder carcinoma. Surg Endosc 20: 721-725, 2006.

94. He RQ, Zhou XG, Yi QY, Deng CW, Gao JM, Chen G and Wang QY: Prognostic signature of alternative splicing events in bladder urothelial carcinoma based on spliceseq data from 317 cases. Cell Physiol Biochem 48: 1355-1368, 2018.

95. Lin P, He RQ, Huang ZG, Zhang R, Wu HY, Shi L, Li XJ, Li Q, Chen $\mathrm{G}$, Yang $\mathrm{H}$ and $\mathrm{He} \mathrm{Y}$ : Role of global aberrant alternative splicing events in papillary thyroid cancer prognosis. Aging (Albany NY) 11: 2082-2097, 2019.

96. Zhang D, Duan Y, Cun J and Yang Q: Identification of prognostic alternative splicing signature in breast carcinoma. Front Genet 10: 278, 2019.

This work is licensed under a Creative Commons Attribution-NonCommercial-NoDerivatives 4.0 International (CC BY-NC-ND 4.0) License. 